\title{
Genetic Diversity Analysis of Red Rice from Hani's Terraced Fields in Yunnan Province
}

\author{
Mengli Ma, Yanhong Liu, Hengling Meng and Bingyue $\mathrm{Lu}^{\mathrm{a}}$ \\ College of Life Science and Technology/ Key Laboratory of Crop High Quality and Efficient \\ Cultivation and Security Control of College in Yunnan Province, Honghe University, Yunnan Mengzi \\ 661100, China \\ aCorresponding author: Iby202@126.com
}

Keywords: Red rice; Hani's terraces fields; Genetic diversity.

\begin{abstract}
Genetic diversity is the main source of variability in any crop improvement program. There are abundant rice landraces in Hani's terraces fields in Yunnan, especially red rice resources. A set of 61 red rice landraces were characterized using 78 simple sequence repeat (SSR) markers to study genetic diversity. A total of 499 fragments were amplified, averaging 6.397 alleles per primer pair. The polymorphism information content ranged from 0.115 to 0.836 with an average of 0.593 , the gene diversity index was 0.633 and heterozygosity was estimated as 0.165 . The results showed that these rice landraces have a rich genetic diversity of Hani’s terraces fields in Yunnan.
\end{abstract}

\section{Introduction}

Rice (Oryza sativa L.) is the most essential and important food crop of the world, particularly in Asia. Genetic diversity is an important basis for rice genetic improvement. Yunnan province is one of the world's recognized centers of genetic diversity and origin of cultivated rice in Asia, is the largest genetic and ecological diversity center of rice germplasm resources in China [1]. The Yuanyang Hani's terraces are located at $22^{\circ} 49^{\prime}-23^{\circ} 19^{\prime} \mathrm{N}, 102^{\circ} 27^{\prime}-103^{\circ} 13^{\prime} \mathrm{E}$. The environment of the uplands is suitable for rice cultivation with average annual sunshine of 1670 hours and an average temperature of $15.4^{\circ} \mathrm{C}$. The Hani's people live on a hillside at an altitude between $1400-2000 \mathrm{~m}$. They have farmed rice in the terraced fields for over 1500 years [2]. A rich diversity of rice varieties thrives there and the local people retain the tradition of seed exchange and rice worship [3]. Grain quality currently represents a major problem in rice production in China and many other rice producing areas of the world [4]. Since a long time ago, Hani's people have believed that the traditional red rice varieties are superior in nutritional quality and healthy effect. The recent studies showed the red rice is superior in antioxidant capacity and nutritional quality $[5,6]$.

Simple Sequence repeat (SSR) markers have been commonly used in genetic diversity studies in rice because of high level of polymorphism which helps to establish the relationship among the individuals even with less number of markers [7]. Previous reports on the genetic diversity of rice using SSR markers showed that genetic diversity was the highest among wild relatives, followed by landraces, and was the lowest for cultivars [8]. The genetic diversity is higher for indica rice compared to japonica rice [9], while the differentiation of cultivated rice is mainly between indica and japonica rice. In recent years, SSR markers have been used to analyze the genetic diversity of Yunnan rice [10-16]. The objectives of the study were to: (1) evaluate the genetic diversity among 61 rice landraces in Hani's terraces fields in Yunnan using SSR markers; 2) provide reference to the rice breeding, germplasm protection.

\section{Materials and Methods}

\subsection{Rice materials}

A collection consisting of 61 red rice landraces was used in this study, which collected from twelve different township of Yuanyang County, Yunnan Province, China as well as from Daping Township, Eza Township, Ganiang Township, Huangcaoling Township, Huangmaoling Township, 
Niujiaozhai Township, Panzhihua Township, Shalatuo Township, Shangxincheng Township, Shengcun Township, Xiaoxinjie Township, Xinjie Town, 12 varieties origin is unknown (Table 1).

Table 1 Name and collecting sites of red rice landraces in this study.

\begin{tabular}{|c|c|c|}
\hline Name of red rice landraces & $\begin{array}{c}\text { Number of } \\
\text { Varieties }\end{array}$ & Collection site \\
\hline cesuogu, honggu2 ,honggu3 & 3 & Daping Township \\
\hline dalonggu, maichou, helingu, wupucheni & 4 & Eza Township \\
\hline honggu1 & 1 & Ganiang Township \\
\hline laojinghongmi, shiouqian, chenilongge, apenpenche & 4 & Huangcaoling Township \\
\hline anxinggu, huagu1, shuihangu & 3 & Huangmaoling Township \\
\hline rongrenche,rongrenrenche, luoge, chemanche, maoche & 5 & Niujiaozhai Township \\
\hline laopinzhonghongmi, laopinzhong & 2 & Panzhihua Township \\
\hline $\begin{array}{c}\text { dianmeng2hao, honggunuo } \\
\text { bendihonggu2, laomujigu }\end{array}$ & 2 & Shalatuo Township \\
\hline chenu, huagu2 & 6 & Shangxincheng Township \\
\hline $\begin{array}{c}\text { bianhao3, poxinggu } \\
\text { baijiaolaojing, yingu, lvjiaogu, longshanlinggu, } \\
\text { aizheranche, chefu, liuyuegu, cheran, chejiagu, } \\
\text { yuelianggu }\end{array}$ & 2 & Shengcun Township \\
\hline $\begin{array}{c}\text { chenal, chemaouzheng, chejiaojiaoer, bendihonggu1, } \\
\text { gutianche, gaoshanhonggu2, pozhugu, chengke, } \\
\text { jiagu,chengni, cheniu, zajiaohonggu }\end{array}$ & 12 & Xiaoxinjie Township \\
\hline
\end{tabular}

\subsection{DNA template preparation and PCR}

DNA was extracted using a protocol adapted from Dellaporta et al. [17]. Following solubilization of the DNA in TE buffer, its concentration was assessed spectrophotometrically, and this was used to prepare working solutions of $20 \mathrm{ng} / \mathrm{ul}$. The PCR analysis was carried out based on SSR primers documented in the Gramene database (www.gramene.org), and the amplification protocols followed Chen et al. [18] with only minor modifications. Briefly, each $10 \mu 1$ reaction contained $10 \mathrm{ng}$ template DNA, $0.2 \mu \mathrm{M}$ of each primer, $2.5 \mathrm{mM}$ dNTP, $1 \mu 110 \mathrm{x} \mathrm{Mg}^{2+}$ free buffer, $25 \mathrm{mM} \mathrm{MgCl}_{2}$ and $0.5 \mathrm{U}$ rTaq DNA polymerase (Sangon Biotech, $\mathrm{CHN}$ ). The cycling regime consisted of a denaturation step $\left(95^{\circ} \mathrm{C} / 5 \mathrm{~min}\right)$, followed by 30 cycles of $95^{\circ} \mathrm{C} / 30 \mathrm{~s}, 55-58^{\circ} \mathrm{C} / 30 \mathrm{~s}, 72^{\circ} \mathrm{C} / 30 \mathrm{~s}$, and a final extension step of $72^{\circ} \mathrm{C} / 10 \mathrm{~min}$. The amplicons were electrophoretically separated through $8 \%$ non-denaturing polyacry lamide gels, and visualized by silver staining [19].

\subsection{Data analysis}

Amplified fragments of different sizes were considered as different alleles. DNA bands that were amplified by a given primer were scored as present (1) or absent (0) for all the samples under study. The average number of alleles per locus, gene diversity, heterozygosity and polymorphism information content (PIC) were calculated using PowerMarker V3.25 [20].

\section{Results}

The results of polymorphism analysis for 61 red rice from Hani's terraced fields in Yunnan Province using 78 SSR markers are shown in Table 2. A total of 499 alleles were detected, and the number of alleles per pair of primers ranged from 2 to 12 with an average value of 6.397. Among the SSR markers, RM481, RM297, RM335, RM7288, RM232, RM444 and OSR28 produced the highest number of alleles, which were 12,11,11,10,10, 10 and 10, respectively; while RM495 produced the smallest number of alleles, which was 2 . The polymorphism information content (PIC) values ranged from 0.115 (RM495) to 0.836 (RM444) with an average of 0.593 , the gene diversity (GD) ranged from 0.123 (RM495) to 0.853 (RM444) with an average of 0.633 . Maximum heterozygosity (H) was 
present at RM311 loci (0.885) and average heterozygosity across all 36 loci was 0.165, RM495, RM138, RM282, RM564, RM258, RM496 loci heterozygosity was 0, heterozygosity was found to be very low which may be due to autogamous nature of rice.

Table 2 Genetic diversity parameter values of SSR (simple sequence repeat) loci.

\begin{tabular}{|c|c|c|c|c|c|c|c|c|c|c|c|}
\hline Marker & Chr. & $\mathrm{NA}$ & GD & $\mathrm{H}$ & PIC & Marker & Chr. & NA & GD & $\mathrm{H}$ & PIC \\
\hline RM495 & 1 & 2.000 & 0.123 & 0.000 & 0.115 & RM3 & 6 & 6.000 & 0.665 & 0.033 & 0.619 \\
\hline RM84 & 1 & 6.000 & 0.773 & 0.082 & 0.737 & RM275 & 6 & 7.000 & 0.666 & 0.049 & 0.614 \\
\hline RM5496 & 1 & 9.000 & 0.829 & 0.049 & 0.808 & RM412 & 6 & 3.000 & 0.500 & 0.098 & 0.424 \\
\hline RM23 & 1 & 8.000 & 0.769 & 0.164 & 0.732 & RM481 & 7 & 12.000 & 0.764 & 0.852 & 0.745 \\
\hline RM493 & 1 & 6.000 & 0.678 & 0.066 & 0.642 & RM125 & 7 & 5.000 & 0.313 & 0.033 & 0.293 \\
\hline RM9 & 1 & 5.000 & 0.517 & 0.016 & 0.479 & RM346 & 7 & 6.000 & 0.594 & 0.066 & 0.516 \\
\hline RM297 & 1 & 11.000 & 0.707 & 0.836 & 0.684 & RM336 & 7 & 6.000 & 0.720 & 0.115 & 0.669 \\
\hline RM14 & 1 & 8.000 & 0.841 & 0.541 & 0.821 & RM478 & 7 & 3.000 & 0.638 & 0.115 & 0.562 \\
\hline RM109 & 2 & 3.000 & 0.320 & 0.016 & 0.291 & RM248 & 7 & 8.000 & 0.820 & 0.066 & 0.796 \\
\hline RM8 & 2 & 4.000 & 0.520 & 0.164 & 0.428 & RM506 & 8 & 6.000 & 0.659 & 0.033 & 0.598 \\
\hline RM7288 & 2 & 10.000 & 0.724 & 0.148 & 0.690 & RM25 & 8 & 6.000 & 0.554 & 0.049 & 0.515 \\
\hline RM8254 & 2 & 9.000 & 0.758 & 0.820 & 0.733 & RM339 & 8 & 9.000 & 0.762 & 0.082 & 0.736 \\
\hline RM475 & 2 & 5.000 & 0.238 & 0.033 & 0.228 & RM80 & 8 & 9.000 & 0.819 & 0.180 & 0.796 \\
\hline RM263 & 2 & 7.000 & 0.755 & 0.131 & 0.715 & RM433 & 8 & 6.000 & 0.691 & 0.033 & 0.641 \\
\hline RM240 & 2 & 6.000 & 0.494 & 0.098 & 0.463 & RM281 & 8 & 6.000 & 0.641 & 0.016 & 0.579 \\
\hline RM138 & 2 & 4.000 & 0.183 & 0.000 & 0.177 & RM444 & 9 & 10.000 & 0.853 & 0.066 & 0.836 \\
\hline RM60 & 3 & 3.000 & 0.332 & 0.082 & 0.287 & RM219 & 9 & 8.000 & 0.813 & 0.262 & 0.793 \\
\hline RM36 & 3 & 6.000 & 0.551 & 0.098 & 0.516 & RM342 & 9 & 6.000 & 0.698 & 0.197 & 0.646 \\
\hline RM218 & 3 & 5.000 & 0.555 & 0.131 & 0.483 & RM105 & 9 & 8.000 & 0.567 & 0.033 & 0.539 \\
\hline RM232 & 3 & 10.000 & 0.832 & 0.148 & 0.811 & RM553 & 9 & 6.000 & 0.559 & 0.115 & 0.523 \\
\hline RM282 & 3 & 4.000 & 0.522 & 0.000 & 0.479 & OSR28 & 9 & 10.000 & 0.662 & 0.098 & 0.639 \\
\hline RM7097 & 3 & 4.000 & 0.440 & 0.049 & 0.410 & RM5095 & 10 & 9.000 & 0.841 & 0.049 & 0.823 \\
\hline RM168 & 3 & 4.000 & 0.419 & 0.016 & 0.391 & RM216 & 10 & 4.000 & 0.674 & 0.131 & 0.615 \\
\hline RM570 & 3 & 5.000 & 0.678 & 0.131 & 0.618 & RM311 & 10 & 8.000 & 0.810 & 0.885 & 0.782 \\
\hline RM307 & 4 & 6.000 & 0.776 & 0.148 & 0.742 & RM258 & 10 & 6.000 & 0.603 & 0.000 & 0.567 \\
\hline RM335 & 4 & 11.000 & 0.801 & 0.721 & 0.780 & RM228 & 10 & 7.000 & 0.706 & 0.098 & 0.656 \\
\hline RM261 & 4 & 4.000 & 0.426 & 0.049 & 0.360 & RM496 & 10 & 5.000 & 0.528 & 0.000 & 0.476 \\
\hline RM471 & 4 & 4.000 & 0.575 & 0.082 & 0.503 & RM286 & 11 & 6.000 & 0.677 & 0.049 & 0.623 \\
\hline RM564 & 4 & 6.000 & 0.570 & 0.000 & 0.503 & RM332 & 11 & 5.000 & 0.630 & 0.066 & 0.563 \\
\hline RM303 & 4 & 6.000 & 0.728 & 0.033 & 0.688 & RM287 & 11 & 6.000 & 0.657 & 0.115 & 0.607 \\
\hline RM122 & 5 & 4.000 & 0.682 & 0.098 & 0.626 & RM21 & 11 & 7.000 & 0.677 & 0.197 & 0.654 \\
\hline RM413 & 5 & 6.000 & 0.783 & 0.115 & 0.750 & RM206 & 11 & 9.000 & 0.834 & 0.066 & 0.817 \\
\hline RM169 & 5 & 5.000 & 0.684 & 0.115 & 0.630 & RM224 & 11 & 4.000 & 0.586 & 0.033 & 0.533 \\
\hline RM163 & 5 & 6.000 & 0.372 & 0.033 & 0.347 & RM20 & 12 & 7.000 & 0.719 & 0.738 & 0.681 \\
\hline RM7415 & 5 & 4.000 & 0.537 & 0.836 & 0.431 & RM19 & 12 & 7.000 & 0.652 & 0.016 & 0.588 \\
\hline RM480 & 5 & 6.000 & 0.424 & 0.082 & 0.402 & RM519 & 12 & 6.000 & 0.692 & 0.066 & 0.649 \\
\hline RM540 & 6 & 6.000 & 0.648 & 0.197 & 0.616 & RM7102 & 12 & 7.000 & 0.746 & 0.508 & 0.712 \\
\hline RM204 & 6 & 9.000 & 0.629 & 0.049 & 0.601 & RM235 & 12 & 9.000 & 0.798 & 0.607 & 0.771 \\
\hline $6-66$ & 6 & 9.000 & 0.816 & 0.115 & 0.791 & RM17 & 12 & 5.000 & 0.555 & 0.131 & 0.509 \\
\hline Mean & & & & & & & & 6.397 & 0.633 & 0.165 & 0.593 \\
\hline
\end{tabular}

Chr., Chromosome; AN, Number of alleles; GD, Gene Diversity; H, Heterozygosity; PIC, Polymorphism information content. 


\section{Discussion}

A clear understanding of genetic diversity and relationships among cultivars using molecular markers is essential for the effective conservation and utilization of rice genetic germplasm resources, which is an important component in the development of improved rice cultivars [21]. In the present study, 78 polymorphic markers have detected a total of 499 alleles across 61 individuals. The number of alleles ranged from 2 in RM495 to 12 in RM481 and the average was 6.397 alleles per SSR locus, which exceeded the previously reported values of 2.161[10], 3.9167[22], 5.065 5.313[16] and 5.5[15] alleles per locus. The higher number of alleles per locus observed in the present study can be attributed to more SSR markers or the larger number of individuals surveyed than in the previous studies. The average PIC value (0.593) exhibited by SSR markers was almost similar to the observed by $\mathrm{Xu}$ et al [16] in 72 current-grown $(0.652)$ and 76 past-grown $(0.660)$ rice landraces, higher averages of PIC value (0.256) were reported by Liu et al. [10]. Among the 78 polymorphic SSR markers, 66 exhibited Gene diversity (GD) higher than 0.500, this result also showed that Hani's red rice landraces have rich genetic diversity. Heterozygosity $(0.165)$ was found to be very low which may be due to autogamous nature of rice. The more diverse nature of the red rice landraces may be a reflection of the prevalent diverse agroclimatic conditions of the Hani's terraces [23]. In conclusion, SSR marker based molecular characterization of Hani's red rice landraces revealed that large variation exists among the accessions. This study will also provide a theoretical basis for the protection and utilization of these rice resources.

\section{Acknowledgments}

This paper is funded by Fundamental Research Program of Yunnan Province (2013FZ124), Yunnan Provincial Department of Education Science Research Fund Project (2013Y066) and Honghe University youth backbone of academic training project (2014GG0101).

\section{References}

[1] Zeng Y, Zhang H, Li Z, et al. Evaluation of genetic diversity of rice landraces (Oryza sativa L.) in Yunnan, China[J]. Breeding Science, 2007, 57(2): 91-99.

[2] Wang Q. On the culture of the terraced fields. Yunnan University Press, 1999, 47-50. (In Chinese).

[3] Xu F R, Tang C F, Yu T Q, et al. Diversity of paddy rice varieties from Yuanyang Hani's terraced fields in Yunnan, China[J]. Acta Ecologica Sinica, 2010, 30(12): 3346-3357.

[4] Tan Y F, Li J X, Yu S B, et al. The three important traits for cooking and eating quality of rice grains are controlled by a single locus in an elite rice hybrid, Shanyou 63[J]. Theoretical and Applied Genetics, 1999, 99(3-4): 642-648.

[5] Gunaratne A, Wu K, Li D, et al. Antioxidant activity and nutritional quality of traditional red-grained rice varieties containing proanthocyanidins[J]. Food chemistry, 2013, 138(2): 1153-1161.

[6] Sumczynski D, Kotásková E, Družbíková H, et al. Determination of contents and antioxidant activity of free and bound phenolics compounds and in vitro digestibility of commercial black and red rice (Oryza sativa L.) varieties[J]. Food Chemistry, 2016, 211: 339-346.

[7] McCouch S R, Chen X, Panaud O, et al. Microsatellite marker development, mapping and applications in rice genetics and breeding[J]. Plant molecular biology, 1997, 35(1-2): 89-99.

[8] Ram S G, Thiruvengadam V, Vinod K K. Genetic diversity among cultivars, landraces and wild relatives of rice as revealed by microsatellite markers. J Appl Genet, 2007, 48(4): 337-345.

[9] ZHANG L, CAO G, HAN L. Genetic diversity of rice landraces from lowland and upland accessions of China[J]. Rice Science, 2013, 20(4): 259-266.

[10]Liu C C, Zhao F W, Wu X X, et al. Genetic diversity and population structure analysis of currently cultivated rice landraces from Hani's Terraced Fields in Yunnan Province moleal. Chin J Rice Sci, 2015, 29(1):28-34. (in Chinese with English abstract) 
[11]Zhang H, Sun J, Wang M, et al. Genetic structure and phylogeography of rice landraces in Yunnan, China, revealed by SSR[J]. Genome, 2006, 50(1): 72-83.

[12]Zhu M Y, Wang Y Y, Zhu Y Y, et al. Estimating genetic diversity of rice landraces from Yunnan by SSR assay and its implication for conservation[J]. Acta Bot Sin, 2004, 46(12): 1458-1467.

[13] Tu M, Lu B R, Zhu Y, et al. Abundant within-varietal genetic diversity in rice germplasm from Yunnan Province of China revealed by SSR fingerprints[J]. Biochemical genetics, 2007, 45(11-12): 789-801.

[14]Zeng Y, Zhang H, Li Z, et al. Evaluation of genetic diversity of rice landraces (Oryza sativa L.) in Yunnan, China[J]. Breeding Science, 2007, 57(2): 91-99.

[15] Bai X H, Gao D, Yu Lei, et al. Genetic diversity of rice landraces from Yuanyang Hani's terraced wetland in Yunnan, China, Molecular Plant Breeding, 2012, 10(3): 1017-1024. (in Chinese with English abstract)

[16] Xu F R, Dong C, Yang W Y, et al. Comparison of genetic diversity of rice landraces planted in two periods in Hani's terraced fields in Yuanyang County, Yunnan Province, China Using Microsatellite Markers[J]. Chinese Journal of Rice Science, 2011, 25(4): 381-386.

[17]Dellaporta SL, Wood J, Hicks JB (1983) A plant DNA minipreparation: Version II. Plant Mol Biol Rep 1:19-21.

[18]Chen X, Temnykh S, Xu Y, et al. Development of a microsatellite framework map providing genomewide coverage in rice (Oryza sativa L.). Theor Appl Genet, 1997, 95:553-567.

[19] Sanguinetti CJ, Dias NE, Simpson AJG. Rapid silver staining and recover of PCR products separated on polyacrylamide gels. Biotechnology,1994, 17:915-919.

[20]Liu K, Muse SV. PowerMarker: an integrated analysis environment for genetic marker analysis. Bioinformatics, 2005, 21(9):2128-2129

[21] Xu Q, Chen H, Wang C H, et al. Genetic diversity and structure of new inbred rice cultivars in China. Journal of Integrative Agriculture, 2012, 11(10): 1567-1573.

[22] Gao D, Li R, Yang M Q, et al. Rice landraces' genetic diversity of Hani terrace wetland in Qingkou village of Yuanyang county. Molecular Plant Breeding, 2011, 9 (118): 1857-1863. (in Chinese with English abstract)

[23] He X H, SunY, Gao D, et al. Comparison of agronomic traits between rice landraces and modern varieties at different altitudes in the paddy fields of Yuanyang terrace, Yunnan province[J]. Journal of Resources and Ecology, 2011, 2(1): 46-50. 